\title{
Dual-Energy CT in Enhancing Subdural Effusions that Masquerade as Subdural Hematomas: Diagnosis with Virtual High-Monochromatic (190-keV) Images
}

\author{
(D) U.K. Bodanapally, DD. Dreizin, (D) G. Issa, DK.L. Archer-Arroyo, (D) K. Sudini, and DT.R. Fleiter
}

\begin{abstract}
BACKGROUND AND PURPOSE: Extravasation of iodinated contrast into subdural space following contrast-enhanced radiographic studies results in hyperdense subdural effusions, which can be mistaken as acute subdural hematomas on follow-up noncontrast head CTs. Our aim was to identify the factors associated with contrast-enhancing subdural effusion, characterize diffusion and washout kinetics of iodine in enhancing subdural effusion, and assess the utility of dual-energy CT in differentiating enhancing subdural effusion from subdural hematoma.
\end{abstract}

MATERIALS AND METHODS: We retrospectively analyzed follow-up head dual-energy CT studies in 423 patients with polytrauma who had undergone contrast-enhanced whole-body CT. Twenty-four patients with enhancing subdural effusion composed the study group, and 24 randomly selected patients with subdural hematoma were enrolled in the comparison group. Postprocessing with syngo.via was performed to determine the diffusion and washout kinetics of iodine. The sensitivity and specificity of dual-energy CT for the diagnosis of enhancing subdural effusion were determined with 120-kV, virtual monochromatic energy (190-keV) and virtual noncontrast images.

RESULTS: Patients with enhancing subdural effusion were significantly older (mean, 69 years; $95 \% \mathrm{Cl}, 60-78$ years; $P<.001$ ) and had a higher incidence of intracranial hemorrhage $(P=.001)$. Peak iodine concentration in enhancing subdural effusions was reached within the first 8 hours of contrast administration with a mean of $0.98 \mathrm{mg} / \mathrm{mL}(95 \% \mathrm{Cl}, 0.81-1.13 \mathrm{mg} / \mathrm{mL})$, and complete washout was achieved at 38 hours. For the presence of a hyperdense subdural collection on 120-kV images with a loss of hyperattenuation on 190-keV and virtual noncontrast images, when considered as a true-positive for enhancing subdural effusion, the sensitivity was $100 \%$ ( $95 \% \mathrm{Cl}, 85.75 \%-100 \%)$ and the specificity was $91.67 \%$ (95\% Cl, 73\%-99\%).

CONCLUSIONS: Dual-energy CT has a high sensitivity and specificity in differentiating enhancing subdural effusion from subdural hematoma. Hence, dual-energy CT has a potential to obviate follow-up studies.

ABBREVIATIONS: $\mathrm{DECT}=$ dual-energy $\mathrm{CT} ; \mathrm{ESDE}=$ enhancing subdural effusion; $\mathrm{HU}=$ Hounsfield unit; $\mathrm{SDH}=$ subdural hematoma; $\mathrm{SECT}=$ single-energy $\mathrm{CT}$; $\mathrm{VNC}=$ virtual noncontrast

D iffusion of contrast material into the subdural space following intravascular contrast administration can result in hyperdense enhancing subdural effusions (ESDEs) on follow-up noncontrast head CTs. ${ }^{1,2}$ These effusions can be mistaken for subdural hematomas (SDHs). ${ }^{1,2}$ Three case reports have previously described ESDEs, all following intra-arterial

Received March 12, 2017; accepted after revision May 25

From the Department of Diagnostic Radiology and Nuclear Medicine (U.K.B., D.D., G.I., K.L.A.-A., T.R.F.), R Adams Cowley Shock Trauma Center, University of Maryland Medical Center, Baltimore, Maryland; and Department of Environmental Health Sciences (K.S.), Bloomberg School of Public Health, Johns Hopkins University, Baltimore, Maryland.

Please address correspondence to Thorsten R. Fleiter, MD, 22 S. Greene St, Department of Radiology, University of Maryland Medical Center, Baltimore, MD 21201 e-mail: tfleiter@gmail.com

Indicates article with supplemental on-line photos.

http://dx.doi.org/10.3174/ajnr.A5318 contrast administration during conventional angiography with resolution documented on short-term follow-up CT examinations. $^{1,2}$

We have frequently observed ESDEs in our busy level 1 trauma center, where patients usually undergo admission contrast-enhanced whole-body CT followed by serial noncontrast head CTs for documented or suspected traumatic brain injury. Because ESDEs can be mistaken for SDHs, lack of awareness of this entity can potentially result in needless delays in instituting thromboprophylaxis and trigger unnecessary follow-up CT studies. Patients with polytrauma usually require thromboprophylaxis to prevent deep vein thrombosis. A number of authors have posited a mandatory 24- to 72-hour period of documented stability of intracranial bleeds before beginning thromboprophylaxis. ${ }^{3-6}$ Hence, early discrimination of SDHs from ESDEs has important clinical implications. 


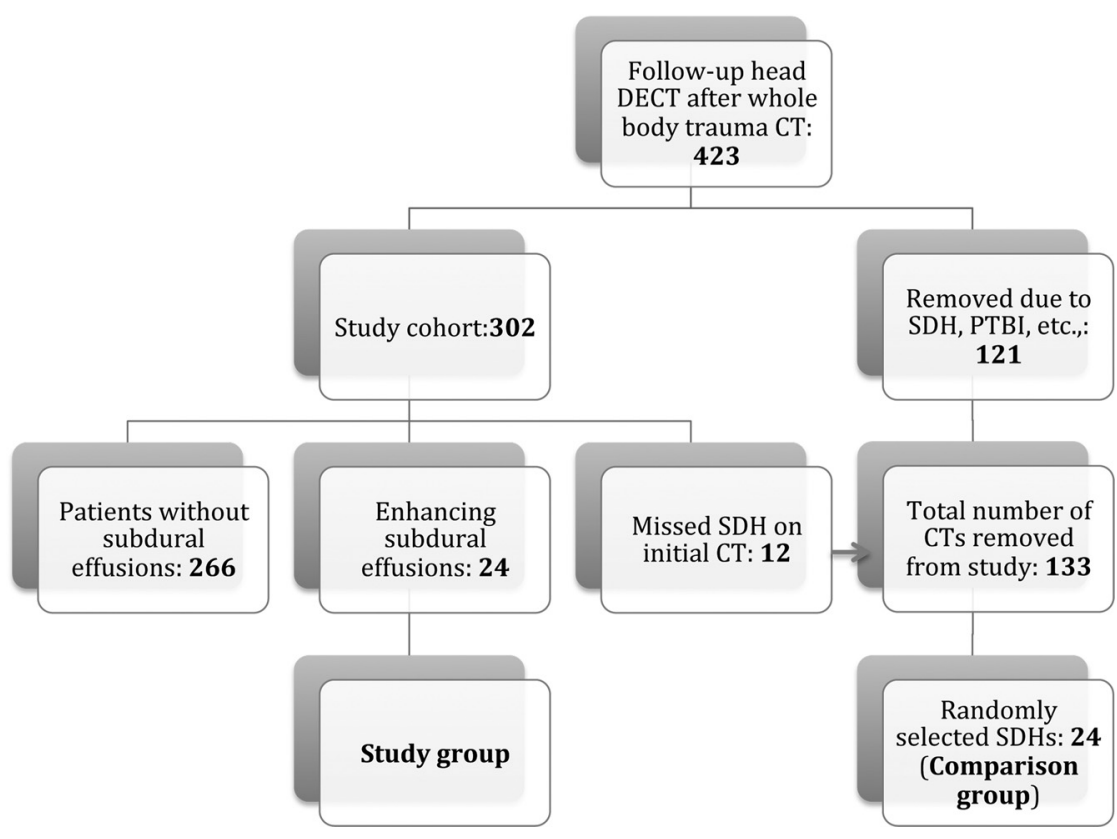

FIG 1. Flowchart shows the patient-selection process. PTBI indicates penetrating traumatic brain injury.

On single-energy CT (SECT), the hyperattenuation caused by hemorrhage and contrast medium is difficult to distinguish due to overlapping Hounsfield units (HU). ${ }^{1,2,7,8}$ Present recommendations for differentiating SDH from ESDE involve serial follow-up imaging. ${ }^{1,2}$ ESDEs shows rapid washout of contrast, hence decreasing hyperattenuation, while SDH retains hyperattenuation from blood for 2-3 weeks. ${ }^{1,2,9}$ Dual-energy CT (DECT) can potentially obviate follow-up scans by differentiating iodine from hemorrhage. ${ }^{7,10,11}$ Iodine overlay maps and virtual noncontrast (VNC) images can discriminate contrast and hemorrhage with a high degree of accuracy. ${ }^{11}$ If VNC images can be used to reliably identify hematoma, even in the presence of iodine, differentiation between ESDEs and SDHs can be a simple and straightforward task. The utility of DECT in diagnosing ESDE was recently demonstrated in a case in which a subdural hyperdense collection that developed after endovascular treatment of an intracranial aneurysm was hyperdense on iodine-overlay images and hypodense on VNC images. ${ }^{12}$ This evidence suggests that DECT may play a vital role in providing an early definitive diagnosis without the need for follow-up CT studies to document resolution of ESDEs.

The purpose of this study was to identify the factors associated with ESDE, characterize the diffusion and washout kinetics of iodine in ESDE, and assess the utility of DECT in differentiating ESDE from SDH.

\section{MATERIALS AND METHODS}

This retrospective study was Health Insurance Portability and Accountability Act-compliant, and permission was obtained from our institutional review board. Informed consent was waived. The study was conducted at a high-volume level 1 trauma center. The inclusion criteria were the following: 1) a history of blunt trauma with acquisition of contrast-enhanced whole-body CT at the time of admission between May 15, 2016, and November 10, 2016;2) acquisition of follow-up noncontrast DECT of the head within 72 hours of the admission CT; 3 ) a radiology report describing a new SDH or hyperdense subdural collection on follow-up head CT; and 4) patients 18 years of age or older. Patients were excluded for the following reasons: 1) There was an SDH on the admission CT; 2) the mechanism of injury was penetrating rather than blunt trauma; and 3) craniectomy, craniotomy, ventricular drain, or pressure-monitoring-device placement was performed, violating the integrity of the meninges.

\section{Subjects}

A search of our Radiology Information System data base from the designated time period yielded 423 patients with at least 1 follow-up head CT performed with DECT within 72 hours of the initial study. At our institution, patients with intracranial injuries, persistent altered mental status, and on anticoagulation without intracranial injuries tend to be evaluated by follow-up head CT, at the discretion of the neurosurgery team. A radiology resident reviewed the head CT reports from this patient cohort for specific data, which included type of intracranial bleed, age, sex, mechanism of injury, and list of interventional procedures. There were 121 patients with SDHs on the initial study or meningeal disruption caused by either penetrating head injuries or interventional procedures performed before a follow-up head DECT was obtained. These patients were excluded from the cohort. The mean age of the final cohort consisting of 302 patients was 47 years (95\% CI, 45-50 years), with 211 men and 91 women. The reports identified 36 patients with new SDHs or hyperdense subdural collections on the first follow-up study. All head CT studies of these 36 patients were further reviewed by a radiologist (reviewer R1) with 8 years of experience to exclude patients with missed SDHs on initial CT as well as hyperdense subdural collections that failed to completely washout on subsequent studies.

Hyperdense subdural collections that failed to show complete washout of contrast were considered enhancing SDHs. Enhancing SDHs show partial washout with clearance of the density contributed by iodine and retain the density from blood on follow-up studies. Twelve such patients with SDHs were excluded from the study group. The remaining 24 patients who had ESDEs on follow-up with subsequent washout of contrast constituted the study group. A 75-year-old woman with posttraumatic SAH developed ESDE after infusion of $250 \mathrm{~mL}$ of contrast media (iohexol, Omnipaque 240 and Omnipaque 300; GE Healthcare, Piscataway, New Jersey) for cerebral angiography, but not after whole-body CT with $100 \mathrm{~mL}$ of Omnipaque 300, and was therefore excluded from the study. Figure 1 shows the patientselection flowchart. An equal number of patients with SDH on 

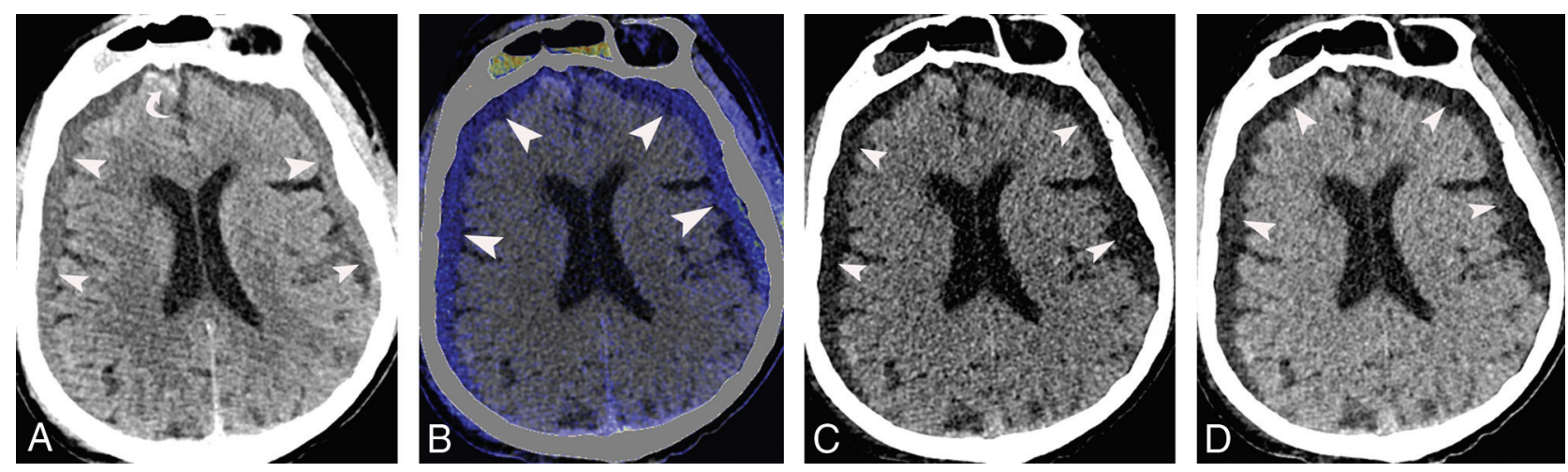

FIG 2. A 75-year-old woman who developed bilateral enhancing subdural effusion on a follow-up study obtained 11 hours after contrast infusion. Axial 120-kV image shows hyperdense subdural effusions (arrowheads, A) and right frontal subarachnoid hemorrhage (curved arrow, B). lodine-overlay image shows contrast-stained subdural effusions (arrowheads). Virtual high-monochromatic (190-keV) (C) and virtual noncontrast $(D)$ images show hypoattenuation (arrowheads) in the fluid.

follow-up DECT were randomly selected from our data base as a comparison group to measure the sensitivity and specificity of DECT in diagnosing ESDE.

\section{Reference Standard}

The presence of ESDE was determined with initial and follow-up images. Absence of SDH on the initial study, evolution of new hyperdense subdural collection on first follow-up CT (Fig $2 A$ and On-line Fig $1 A$ ), and subsequent washout of hyperattenuation in follow-up studies were used as evidence for ESDE. ${ }^{1,2,12}$

\section{Imaging Technique and Contrast Medium Injection}

Admission whole-body CT examinations were performed on a DECT (Somatom Force; Siemens) or 64-channel SECT scanner (Brilliance; Philips Healthcare, Best, the Netherlands). Wholebody CT involves a noncontrast head CT followed by contrastenhanced CT of the neck, chest, abdomen, and pelvis. The studies were performed after injection of $100 \mathrm{~mL}$ of iodinated contrast media (Omnipaque 350) with a split bolus, with $60 \mathrm{~mL}$ injected at $5 \mathrm{~mL} / \mathrm{s}$ and $40 \mathrm{~mL}$ injected at $4 \mathrm{~mL} / \mathrm{s}$. The contrast injection was followed by a $50-\mathrm{mL}$ saline injection at $4 \mathrm{~mL} / \mathrm{s}$.

Follow-up head CT studies were performed with a DECT or SECT scanner, depending on scanner availability. DECT images were obtained with the $\mathrm{x}$-ray tubes at $80 \mathrm{kV}$ and Sn150 kV. "Sn" denotes the use of additional tin filter that increases the mean photon energy of the respective spectrum. Scan parameters were as follows: rotation time, 0.5 seconds; pitch, 0.55 . The reference milliampere-second was $273 \mathrm{mAs}$ for the Sn150-kV and $410 \mathrm{mAs}$ for the $80-\mathrm{kV}$ tube. Original dual-energy datasets were reconstructed with an increment of $1 \mathrm{~mm}$ and section thicknesses of 1and 5-mm for the 120-kV-equivalent mixed DECT images with an adaptive iterative reconstruction algorithm (ADMIRE, Siemens) with strength levels of 2 and 5, respectively. Automatic tube current modulation (CARE Dose4D; Siemens) was used in all patients. The mean CT dose index volume and dose-length product were $31.45 \pm 2.95 \mathrm{mGy}$ and $609.62 \pm 76.79 \mathrm{mGy} \times \mathrm{cm}$, respectively.

\section{Image Analysis of DECT}

For measuring the diffusion and washout kinetics of iodine into the subdural effusions, all of the follow-up dual-energy datasets for head CTs from each patient, performed within 72 hours of the admission scan, were transferred to a postprocessing workstation (syngo.via, version VB10B; Siemens) to quantify iodine in the subdural space. If we included the first follow-up DECT data from the 24 patients, there were 47 follow-up DECT datasets, which were analyzed for evaluating iodine diffusion and washout kinetics. The maximum number of DECT studies from each patient evaluated was 3. Many of the patients had a combination of DECT and SECT follow-up studies. In such patients, DECT datasets were used for evaluating iodine diffusion and washout kinetics, while all the follow-up studies (both DECT and SECT) were used to evaluate subsequent washout of hyperattenuation to confirm the diagnosis of ESDE. A modified brain hemorrhage application was used, with the bone beam-hardening box checked and changes to the standard parameters in the syngo.via dual-energy configuration of gray-scale ( window level $=31$; width $=54$ ) and dual-energy ( window level $=28$; width $=66$ ), was made for the color look-up table. Similarly, the material decomposition algorithm was modified into hemorrhage, 80/75 HU (80 kV/Sn150 $\mathrm{kV})$; CSF, 16/5 HU ( $80 \mathrm{kV} / \mathrm{Sn} 150 \mathrm{kV})$; and iodine, with the relative contrast medium adjusted to a value of 3.46 .

Reviewer R1 performed postprocessing of the data. From the original low- and high-kilovolt datasets, a VNC series and functional and quantitative iodine series were derived. In the functional images, the presence of iodine was indicated by blue in a special color-coded look-up table, with intensity corresponding to the relative concentration of iodine (Fig 2B). The software allows quantitative analysis of ROIs. After selection on the iodine image, the software calculated the concentration of iodine in milligrams/milliliter and the ROI size in square millimeters.

This algorithm also calculates the attenuation (HU) values from VNC and virtual 120-kV images and iodine-related attenuation. On each side of the cerebral convexities, 3 different ROIs of $0.2 \mathrm{~cm}^{2}$ were used at different image sections. To promote uniformity in the measurements, we used approximately the same anatomic locations for the ROIs on sequential follow-up CT studies. The reviewer was blinded to the color-overlay images to prevent ROI placement over the maximum concentration of contrast medium for measuring the respective values. From the different 

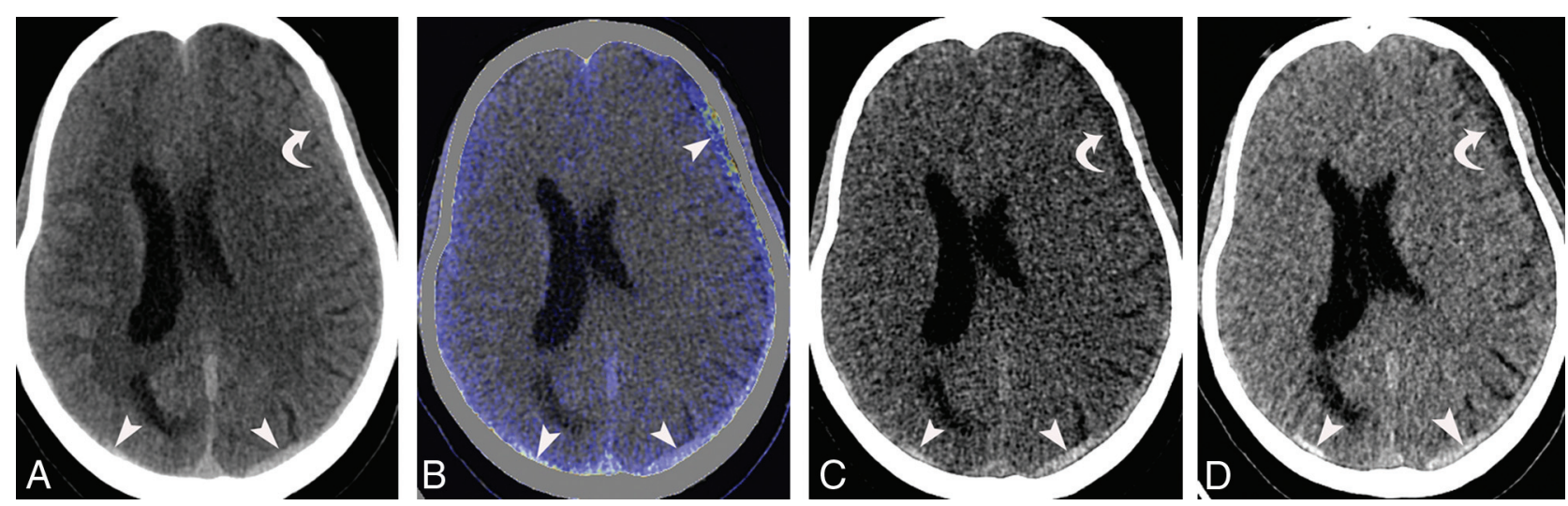

FIG 3. A 55-year-old man with left cerebral convexity and right occipital acute subdural hematomas with enhancement of a left subdural hematoma on a follow-up study obtained 6 hours after contrast infusion. Follow-up axial 120-kV $(A)$ and iodine-overlay (B) images show hyperdense and contrast-stained subdural hematomas, respectively (arrowheads). Virtual high-monochromatic (190-keV) (C) and virtual noncontrast $(D)$ images show hyperattenuation corresponding to the hematoma over the occipital lobes and left temporal lobe (arrowheads) and hypoattenuation corresponding to the enhancing component of hematoma over the left frontal lobe (curved arrow).

Table 1: Comparison of DECT findings differentiating ESDE from SDH

\begin{tabular}{cccll}
\hline Diagnosis & $\begin{array}{c}\text { Traditional Mixed } \\
(\mathbf{1 2 0 - k V )} \text { Images }\end{array}$ & $\begin{array}{c}\text { High-Monochromatic } \\
(\mathbf{1 9 0}-\mathrm{keV}) \text { Images }\end{array}$ & VNC Images & $\begin{array}{c}\text { Iodine-Overlay } \\
\text { Images }\end{array}$ \\
\hline ESDE & Hyperdense & Hypodense & Hypodense & Positive \\
SDH & Hyperdense & Hyperdense & Hyperdense & Positive/negative \\
\hline
\end{tabular}

associations were determined by regression analysis. One-way analysis was used to determine the iodine concentrations, and contingency tables were used to determine sensitivity and specificity. $\kappa$ statistics were used to test interobserver re-

ROIs, the mean iodine concentration and VNC-, mixed 120-kV-, and iodine-related attenuation were calculated. At the same time, the maximum thickness of the effusion was measured.

Two experienced radiologists performed DECT image analysis, reviewer R2 with 8 years and reviewer R3 with 5 years of experience. For each of the first follow-up studies performed on the DECT, axial 120-kV (standard gray-scale), 190-keV (virtual monochromatic energy), and VNC images created on a workstation were loaded onto a dedicated work list. Apart from the VNC images, we used $190-\mathrm{keV}$ images due to the theoretic ability to achieve iodine removal because the energy used is far away from the $k$-edge of iodine, thus greatly decreasing the iodine attenuation to negligible values. Blinded to the initial and follow-up head CT findings, each radiologist reviewed the images, interpreting the $120-\mathrm{kV}$ and $190-\mathrm{keV}$ images separately. The studies were assessed for the presence or absence of hyperdense (relative to CSF) subdural collections on each set of images and were scored as nominal variables. In the final step, the VNC images were given as the third set, and reviewers were asked to score the density of subdural collections in a similar fashion. The presence of a hyperdense subdural collection on 120-kV images with loss of hyperattenuation on 190-keV and VNC images is considered a true-positive for ESDE (Fig 2 and On-line Fig 1), while hyperattenuation on all the 3 sets is considered SDH (Fig 3 and On-line Fig 2). Discrepancies between the assessments of the 2 reviewers were resolved by adjudication by another reviewer (R4 with 10 years of experience). Criteria used to differentiate ESDE from SDH are given in Table 1.

\section{Statistical Analysis}

Statistical analysis was performed by K.S., with statistical software (JMP 12; SAS Institute, Cary, North Carolina). Differences between the ESDE group and those without were determined with odds ratios and $\chi^{2}$ and Wilcoxon tests. The strongest independent liability in assessing CT variables by the radiologists and to test the agreement between nominal scores that were derived from the density of subdural collections on $190-\mathrm{keV}$ and VNC images.

\section{RESULTS}

Among the 24 patients with ESDE, there were 18 men and 6 women. The incidence of ESDE in patients with trauma with follow-up head CT was $8.2 \%$. The mean age of the patients with ESDE was 69 years (95\% CI, 60-78 years). Patients with ESDE were significantly older than those without $(P<.001)$, with an incidence of $13 \%$ in patients older than 50 years of age. There were 12 patients with bilateral and 12 with unilateral ESDEs, with a mean thickness of $4.2 \mathrm{~mm}$ (95\% CI, 3.7-4.8 mm). The ESDEs mainly involved the frontal, parietal, and temporal lobes. None of the patients had mass effect or midline shift.

Table 2 shows the differences in the CT findings between patients with ESDE and those without. There was a higher association of ESDE with intracranial hemorrhage $(P=.001)$, specifically with subarachnoid hemorrhage $(P=.002)$. There was no association with cerebral contusions $(P=0.97)$ or CT evidence of diffuse axonal injury $(P=0.33)$. None of the patients with ESDE had epidural hematoma. According to stepwise logistic regression analysis, age was the most reliable predictor of $\operatorname{ESDE}(P<.001)$, followed by the presence of intracranial hemorrhage $(P=.002)$.

\section{Diffusion and Washout Kinetics of lodine}

All 24 patients had their first DECT follow-up from 4 to 24 hours after whole-body CT (median, 7 hours; interquartile range, 4). The peak concentration of iodine in ESDEs was reached in the scans obtained within the first 8 hours of contrast administration, with a mean of $0.98 \mathrm{mg} / \mathrm{mL}$ (95\% CI, $0.81-1.13 \mathrm{mg} / \mathrm{mL})$. The mean value of iodine concentration was 0.71 (95\% CI, $0.48-0.94$ $\mathrm{mg} / \mathrm{mL}$ ) in the scans obtained between 8 and 16 hours, and 0.41 (95\% CI, 0.16-0.67), between 16 and 38 hours, reflecting gradual 
Table 2: CT findings in ESDE and the control groups

\begin{tabular}{lcccc}
\hline \multicolumn{1}{c}{ Finding } & $\begin{array}{c}\text { ESDE Group } \\
(\text { No. })(\boldsymbol{n}=\mathbf{2 4})\end{array}$ & $\begin{array}{c}\text { Non-ESDE Group } \\
(\text { No.) }(\boldsymbol{n}=\mathbf{2 6 6})\end{array}$ & $\begin{array}{c}\text { Odds Ratio } \\
(\mathbf{9 5 \%} \mathrm{CI})\end{array}$ & $\boldsymbol{P}$ Value \\
\hline SAH & 16 & 94 & $3.7(1.52-8.97)$ & .002 \\
Contusions & 7 & 79 & $0.98(0.39-2.46)$ & .97 \\
Diffuse axonal injury & 2 & 11 & $2.12(0.44-10.2)$ & .33 \\
Normal CT findings & 4 & 136 & $5.15(1.7-15.5)$ & .001 \\
\hline
\end{tabular}

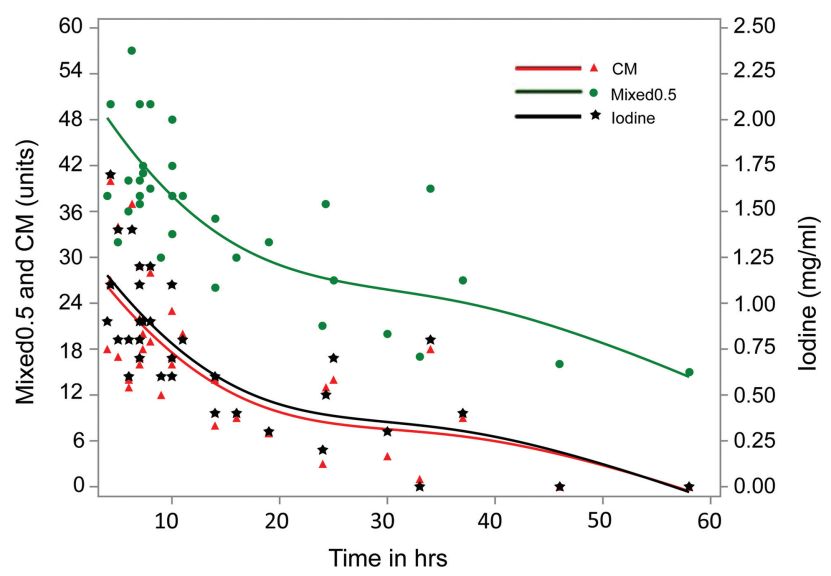

FIG 4. Time-concentration curve. Spline graph using penalized B-spline shows iodine (milligrams/milliliter), attenuation (HU) related to contrast media (CM), and mixed 120-kV (Mixed 0.5) images versus hours (hrs) after contrast administration.

washout. Complete washout was observed after 38 hours (Fig 4). The maximum concentration of iodine found in our group was $1.7 \mathrm{mg} / \mathrm{mL}$ on a study performed after 4.5 hours of contrast administration. None of the studies retained contrast beyond 38 hours. Iodine concentration and iodine-related attenuation had a correlation coefficient of 0.99 (95\% CI, 0.98-0.99). The regression coefficient indicated that for every additional $0.1 \mathrm{mg} / \mathrm{mL}$ of iodine, the iodine-related attenuation increased by an average of 2.4 HU. The maximum attenuation reached in the subdural effusions was $67 \mathrm{HU}$ (median, $35 \mathrm{HU}$; interquartile range, $16 \mathrm{HU}$ ) on mixed $120-\mathrm{kV}$ images. The mean density of the subdural effusions as related to VNC images was found to be $19.98 \mathrm{HU}$ (95\% CI, 18.98-20.98 HU), corresponding to baseline attenuation of subdural effusion. Figure 4 shows the time-concentration curves of iodine with inclusion of attenuation related to contrast media and overall attenuation on mixed $120-\mathrm{kV}$ images.

\section{Specificity of Virtual High-Monochromatic Energy (190-keV) and VNC Images}

All ESDEs were correctly interpreted as hyperdense subdural fluid on mixed 120-kV images and hypodense fluid on 190-keV and VNC images, because 190-keV and VNC images remove iodine. Twenty-two of the 24 SDHs were correctly interpreted as hyperattenuated subdural collections on $120-\mathrm{kV}, 190-\mathrm{keV}$, and VNC images, and the remaining 2 studies were falsely interpreted as ESDE. Both of these were enhancing SDHs. The presence of a subdural collection on $120-\mathrm{kV}$ images with loss of hyperattenuation on 190-keV images, when considered as true-positive for ESDE, had a sensitivity of $100 \%$ (95\% CI, 85.75\%-100\%) and specificity of $91.67 \%$ (95\% CI, 73\%-99\%). There was a perfect agreement $(\kappa=1)$ between $190-\mathrm{keV}$ and VNC images with regard to the nominal scores that were derived to define the density of the subdural collection. There was almost perfect interobserver agreement between the 2 radiologists $(\kappa=0.88 ; 95 \%$ CI, 0.72-1).

\section{DISCUSSION}

We analyzed the factors associated with ESDE and the ability of DECT to discriminate ESDE from SDH in patients with trauma after wholebody CT. According to our results, patients with ESDE were significantly older $(P<.001)$ and tended to have intracranial hemorrhage $(P=.001)$. The results demonstrate that DECT can differentiate ESDE from SDH with high sensitivity and specificity.

Hyperattenuation caused by hemorrhage and contrast medium is difficult to discriminate on SECT because effusions can demonstrate varying densities. ${ }^{1,2,7,8}$ Some patients with ESDE have very high densities (range, 97-200 HU) as opposed to blood (range, 28-82 HU; mean, $54 \mathrm{HU}$ ), and some have lower densities (35 HU), depending on the degree of iodine dilution in the effusion. ${ }^{1,2,8}$ If the attenuation exceeds the value expected for blood, it can be assumed to have an iodine component, but this does not exclude an underlying SDH because contrast is known to diffuse into SDH (Fig 3 and On-line Fig 2). ${ }^{7,13}$ Similarly, iodine overlay maps help in identifying contrast in subdural collections but do not differentiate ESDEs from enhancing SDHs (Fig 3B). ${ }^{7,10}$ Hence, one has to rely on $190-\mathrm{keV}$ or VNC images to identify a hematoma even when mixed with iodine (Fig $3 C$, $-D$ and On-line Fig $2 C,-D)$, to consistently differentiate enhancing $\mathrm{SDH}$ from ESDE.

Virtual high-monochromatic (190-keV) and VNC image reconstruction relies on the same measurement of attenuation differences in an ROI using 2 different effective $x$-ray spectra of a dual-energy scanner. The difference is that the percentage of the attenuation contributed by iodine in each voxel is subtracted or replaced in the VNC images but not in the virtual monochromatic images. Instead the attenuation contribution of the detected material is projected for a given kiloelectron volt in monochromatic images. However, the attenuation contribution of iodine is negligible at $190-\mathrm{keV}$ and therefore should be comparable with the VNC approach of iodine subtraction or virtual replacement. (Fig $2 C,-D$ and On-line Fig $1 C,-D) .{ }^{14,15}$ We have demonstrated that high-monochromatic images can be used in place of VNC images to identify ESDE after excluding iodine from the images. However, there were $2 \mathrm{SDH}$ s that were falsely interpreted as ESDEs. Both of these were enhancing SDHs with a large enhancing component and a smaller SDH component (Fig 3C, -D). The reviewers overlooked the hematoma component on both $190-\mathrm{keV}$ and $\mathrm{VNC}$ images and misinterpreted them. Hence, we recommend radiologists considering an enhancing $\mathrm{SDH}$ before diagnosing ESDE and carefully reviewing the $190-\mathrm{keV}$ and VNC images for possible underlying small SDHs.

The reported cases by Zamora and $\operatorname{Lin}^{1}$ and Rennert and Hamer $^{2}$ followed intra-arterial administration of contrast during angiography with use of $260 \mathrm{~mL}$ of Imeron 300 (iopamidol; Bracco, Milan, Italy) in 1 patient. ${ }^{1,2}$ Our patients differ from those in these previous reports because the contrast was administered intravenously at moderate doses $(100 \mathrm{~mL})$. Zamora and Lin did 
not mention the amount of contrast administered in their 2 patients during cerebral angiography; however, we presume that the contrast used was significantly higher than that of our patients. The reports had 2 patients with a high attenuation of 97 and $200 \mathrm{HU}$ in the effusion and 1 patient with an attenuation of 35 $\mathrm{HU}$, respectively. However, none of our effusions reached the high attenuation previously reported. The maximum attenuation reached in our subjects was $67 \mathrm{HU}$ (median, $35 \mathrm{HU} ; \mathrm{Q}_{3}-$ $\left.\mathrm{Q}_{1}, 16 \mathrm{HU}\right)$. The differences may be due to the lower dose of contrast used in our patients and also the intravenous route of administration.

Rennert and Hamer ${ }^{2}$ proposed that the intra-arterial route of administration was a predisposing factor contributing to ESDEs. We believe that the route of contrast administration likely plays a role along with the amount of contrast used because 1 of our patients developed ESDE after intra-arterial administration of a large dose of contrast $(250 \mathrm{~mL})$ during cerebral angiography, but not after intravenous administration of a moderate contrast dose $(100 \mathrm{~mL})$ during whole-body CT. Zamora and $\mathrm{Lin}^{1}$ proposed that the presence of SAH increases dural endothelial permeability with extravasation of contrast material by triggering a neuroinflammatory response, while Rennert and Hamer ascribed the increase in permeability to osmotic disruption caused by hyperosmolar contrast media. Our results suggest that diffusion of contrast into the subdural space is unlikely to be solely related to the inflammatory theory because this phenomenon is also seen in patients without SAH and in patients without any demonstrable intracranial abnormalities. Furthermore, osmotic disruption may not be an essential element of ESDE development because the capillaries of the dura are naturally fenestrated and allow free passage of contrast media into the dural extracellular space, depending on the hydrostatic pressure, producing enhancement. $^{16}$

On the basis of the results of our study, with age being the strongest predictor, we propose an alternate theory: We postulate that the pathophysiology of ESDE follows that of patients with intracranial hypotension. According to the Monroe-Kellie rule, intracranial volume is constant and is represented by the sum volume of brain substance, CSF, and blood. ${ }^{17,18}$ Therefore, with cerebral volume loss associated with advanced age, there will be a compensatory increase in CSF and intracranial blood volume. The increase in intracranial blood volume manifests in the form of dural vasodilation, greater concentration of contrast in dural vasculature, and subdural effusions. ${ }^{17-21}$ Dural venous engorgement causes leakage of intravascular, nonhemorrhagic fluid across a hydrostatic pressure gradient into the subdural space, carrying contrast with it because the dural vasculature lacks tight junctions, thus not contributing to the blood-brain barrier. ${ }^{17}$ Similar to effusions in intracranial hypotension, enhancing effusions involve frontoparietal areas and are thin $(4.2 \mathrm{~mm}$; $95 \% \mathrm{CI}$, $3.7-4.8 \mathrm{~mm}$ ) without mass effect on the brain. ${ }^{20,22}$ The intrinsic high density of this effusion (mean, $19.98 \mathrm{HU}$; 95\% CI, 18.98$20.98 \mathrm{HU}$ ) on VNC images may be related to the concentration of protein in the fluid, as is seen in subdural fluid in patients with intracranial hypotension. ${ }^{20}$

Finally, we believe that these ESDEs do not represent traumatic subdural hygromas because hygromas tend to develop be- tween 1 and 2 weeks after head injury rather than within the first few days. ${ }^{13,23}$ Moreover, studies have demonstrated that contrast does not influx into subdural hygromas. ${ }^{13}$

\section{Limitations}

Quantification of iodine in the subdural space is limited by noise (eg, scatter). Increasing the radiation dose from our parameters achieved by setting off CARE Dose would have been optimum, but it would have resulted in more radiation. The use of the modified brain hemorrhage protocol in syngo.via in our image analysis might have minimized the errors caused by beam-hardening, the wrong kernel, or any other wrong scan parameters.

\section{CONCLUSIONS}

DECT has high sensitivity and specificity in differentiating ESDE from SDH with a potential to obviate follow-up studies.

\section{REFERENCES}

1. Zamora CA, Lin DD. Enhancing subdural effusions mimicking acute subdural hematomas following angiography and endovascular procedures: report of 2 cases. J Neurosurg 2015;123:1184-87 CrossRef Medline

2. Rennert J, Hamer OW. Large subdural effusions after angiography mimicking acute subdural hematoma. J Comput Assist Tomogr 2010; 34:249-50 CrossRef Medline

3. Farooqui A, Hiser B, Barnes SL, et al. Safety and efficacy of early thromoboembolism chemoprophylaxis after intracranial hemorrhage from traumatic brain injury. J Neurosurg 2013;119:1576-82 CrossRef Medline

4. Phelan HA, WolfSE, Norwood SH, et al. A randomized double-blinded, placebo-controlled pilot trial of anticoagulation in low-risk traumatic brain injury: the Delayed Versus Early Enoxaparin Prophylaxis I (DEEP I) study. J Trauma 2012;73:1434-41 CrossRef Medline

5. Norwood SH, Berne JD, Rowe SA, et al. Early venous thromboembolism prophylaxis with enoxaparin in patients with blunt traumatic brain injury. J Trauma 2008;65:1021-26; discussion 1026-27 Medline

6. Koehler DM, Shipman J, Davidson MA, et al. Is early venous thromboembolism prophylaxis safe in trauma patients with intracranial hemorrhage. J Trauma 2011;70:324-29 CrossRef Medline

7. Gupta R, Phan CM, Leidecker C, et al. Evaluation of dual-energy CT for differentiating intracerebral hemorrhage from iodinated contrast material staining. Radiology 2010;257:205-11 CrossRef Medline

8. Willmann JK, Roos JE, Platz A, et al. Multidetector CT: detection of active hemorrhage in patients with blunt abdominal trauma. AJR Am J Roentgenol 2002;179:437-44 CrossRef Medline

9. Bodanapally UK, Sours C, Zhuo J, et al. Imaging of traumatic brain injury. Radiol Clin North Am 2015;53:695-715, vii CrossRef Medline

10. Phan CM, Yoo AJ, Hirsch JA, et al. Differentiation of hemorrhage from iodinated contrast in different intracranial compartments using dual-energy head CT. AJNR Am J Neuroradiol 2012;33:1088-94 CrossRef Medline

11. Postma AA, Das M, Stadler AA, et al. Dual-energy CT: what the neuroradiologist should know. Curr Radiol Rep 2015;3:16 CrossRef Medline

12. Tan LA, Chen M, Muñoz LF. Letter to the editor: utility of dualenergy CT in differentiating contrast extravasation from intracranial hematoma. J Neurosurg 2016;124:279-80 CrossRef Medline

13. Mori K, Maeda M. Delayed magnetic resonance imaging with GdDDTPA differentiates subdural hygroma and subdural effusion. Surg Neurol 2000;53: 303-10; discussion 310-11 CrossRef Medline

14. Shinohara Y, Sakamoto M, Iwata N, et al. Usefulness of monochromatic imaging with metal artifact reduction software for computed 
tomography angiography after intracranial aneurysm coil embolization. Acta Radiol 2014;55:1015-23 CrossRef Medline

15. Potter CA, Sodickson AD. Dual-energy CT in emergency neuroimaging: added value and novel applications. Radiographics 2016;36:2186-98 CrossRef Medline

16. Sage MR. Kinetics of water-soluble contrast media in the central nervous system. AJNR Am J Neuroradiol 1983;4:897-906 CrossRef Medline

17. Fishman RA, Dillon WP. Dural enhancement and cerebral displacement secondary to intracranial hypotension. Neurology 1993;43: 609-11 CrossRef Medline

18. Paldino M, Mogilner AY, Tenner MS. Intracranial hypotension syndrome: a comprehensive review. Neurosurg Focus 2003;15:ECP2 Medline
19. Brightbill TC, Goodwin RS, Ford RG. Magnetic resonance imaging of intracranial hypotension syndrome with pathophysiological correlation. Headache 2000;40:292-99 CrossRef Medline

20. Mokri B, Parisi JE, Scheithauer BW, et al. Meningeal biopsy in intracranial hypotension: meningeal enhancement on MRI. Neurology 1995;45:1801-07 CrossRef Medline

21. Koss SA, Ulmer JL, Hacein-Bey L. Angiographic features of spontaneous intracranial hypotension. AJNR Am J Neuroradiol 2003;24: 704-06 Medline

22. Pannullo SC, Reich JB, Krol G, et al. MRI changes in intracranial hypotension. Neurology 1993;43:919-26 CrossRef Medline

23. Yamada H, Watanabe T, Murata S, et al. Developmental process of chronic subdural collections of fluid based on CT scan findings. Surg Neurol 1980;13:441-48 Medline 